\title{
用瞬态光致发光研究非对称双量子 阱隧穿过程*
}

\author{
黄旭光 蔡志岗 李庆行 余振新 \\ (中山大学超快速激光光谱学国家重点实验室,广州 510275)
}

王太宏 梅笑冰 杨国桢

(中国科学院物理研究所, 北京 100080)

\section{关链词非对称双贯子陆、隧穿过程、瞬态光致发光}

半导体量子拼超晶格结构的载流子除了具有平行于阱层界面的纵向运动 (纵向输运) 外, 还具有垂直于界面方向的横向运动 (横向输运). 横向运动需要穿过势垒阻挡层, 因此又称为载 流子的隧道穿透效应 (Tunneling, 简称隧穿). 自从 1973 年 Esaki 和 $\mathrm{Tsu}$ 等人的开创性 工作以来, 特别是量子际超晶格材料的问世, 有关隧穿问题引起人们极大的关注. 一方面是出 于物理学自身发展的需要, 对隧穿过程的理解有助于阐明固体中电子波函数的基本特性, 无其 是与时间有关的动态行为. 另一方面是由于隧穿过程决定着所步及元器件的极限响应速度, 对超高速光、电子学器件的研制具有重要意义. 然而过去对隧穿效应的研究, 在理论上, 除了 稳态特性方面外, 在动力学方面反提出了基于波函数相干迭加的相干隧 穿 模 型 (Coherent tunneling) ${ }^{23}$ 和基于散射的序列隧穿模型 (Sequence tunneling) ${ }^{[3]}$. 而在实验上极少涉及到 有关电子或空穴的隧穿动力学特性和机制的研究. 这主要是因为该过程的特征时间尺度只有 皮秒量级, 超出了通常的测量手段和技术的分辨根限. 随着超快速激光光谱学技术的发展, 人 们才有可能从实验上直接研究隧穿的动力学过程. 1986 年, Masumoto 等人 ${ }^{[4]}$ 首次运用时间 纷辨发光技术研究了超晶格结构载流子沿垂直于吽层方向 (即生长方向) 的隧穿过程. 1989 年以后, 由于采用了非对称耦合双量子吽结构 (ADQW), 有关电子或空穴的共振和非共振 隧穿动力学机制的研究才有了很大的进展 ${ }^{[5-9]}$. 目前已有的电子或空穴隧穿实验揭示: (1) 实 验基本排除了相干隧穿机制, 因为碰撞、散射等弛豫过程能够在极短的时间内破坏了波函数 (位相)相干性 ${ }^{[103}$; (2)通过改变外加电场等因素可以调节 $A D Q W$ 结构中两阱的能级差, 从而 控制隧穿的时间或速率, 实验所谓的共振隧穿或非共振隧穿; (3) 杂质, 合金或界面散射以及 LO 声子散射等辅助型序列隧穿是可能的共振以及非共振隧穿机制. 可见, 已有的电子或空 穴隧穿研究工作, 尽管获得了时间值及其与势垒宽度、电场大小等参数关系的信息, 探讨了可 能的隧穿机制, 使人们对隧穿动力学过程有了一定的认识, 但仍存在一些问题有待解决, 无其 是未能就有关的隧穿机制作出明确的令人满意的回答,还有待于作更深入的研究.

本文运用皮秒时间分辨光致发光技术研究了不同中垒厘度和 不 同 掺 杂方式的 GaAs/ $\mathrm{AlGaAs}$ 非对称双量子阱结构电子隧穿动力学过程, 排除了相干隧穿机制和杂质散射辅助型 序列隧穿机制, 并初步提出了一种新的有关电子或空穴共振和非共振隧穿机制。 


\section{1 实 验 部 分}

实验所用的 $\mathrm{GaAs} / \mathrm{AlGaAs} \mathrm{ADQW}$ 样品是采用国产分子束外延技术, 在 $\mathrm{GaAs}$ 衬底上 逐层生长而成,其中有 5 个周期的双量子阱结构 $(100 \AA$ 宽的 $\mathrm{GaAs}$ 宽阱、各种垒厚的 $\mathrm{AlGaAs}$

表 1 样品参数

\begin{tabular}{|c|c|c|}
\hline 样品号 No. & 中垒湈度 $(\AA)$ & 掺杂方式 \\
\hline 40 & 40 & 不掺 杂 \\
\hline 60 & 60 & 两边秝掺杂 \\
\hline 80 & 80 & 两边拍掺杂 \\
\hline 200 & 200 & 两边垒掺杂 \\
\hline 601 & 60 & 中垒不掺杂 \\
\hline 602 & 60 & 中秝 $\delta$ 掺杂 \\
\hline 603 & 60 & 中㖉 $10 \AA$ 掺染 \\
\hline
\end{tabular}

中垒和 $50 \AA$ 宽的 $\mathrm{GaAs}$ 窄阱). 一些样品不故意掺杂, 另一些样品采用各种方式掺杂, 包括两 边垒掺 $\mathrm{Be}\left(1 \times 10^{19} \mathrm{~cm}^{-3}\right)$ 、中垒中部 $10 \AA$ 内掺 $\mathrm{Be}\left(3 \times 10^{18} \mathrm{~cm}^{-3}\right)$ 和中垒 $\delta$ 掺杂等. 详细情 况如表 1 所示.

实验采用皮秒时间分辨光致发光技术. 一台同步泵浦锁模 $\mathrm{Rh} 6 \mathrm{G}$ 染料激光器腔倒空输 出光脉仲列作为激发源, 样品的发光由配有光棚单色仪的同步扫描条纹照相机系统探测记录, 具体实验装置和方法见参考文献 [11], 整个实验系统的时间分辨率约 $10 \mathrm{ps}$. 样品犆于 $77 \mathrm{~K}$ 液 氮致冷器内.

\section{2 结果与讨论}

$A D Q W$ 受到光照射时, 其窄量子阱和宽量子阱的电子均被激发到导带而复合发光. 由 于窄阱 $n=1$ 态的能量高于宽陎相应的能态, 所以窄阱的电子除了复合发光外, 同时还会以一 定的速率 $\tau_{T}^{-1}$ 隧穿到宽阱. 因此, 窄阱发光强度衰减速率 $\tau^{-1}$ 满足关系式 $^{[7}$ :

$$
\tau^{-1}-\tau_{\gamma}^{-1}+\tau_{T}^{-1},
$$

其中 $\tau_{r}$ 为复合寿命. 基于公式 (1) 的关系, 我们可以由窄阱的时间分辨光致发光衰减过程来 研究电子从窄阱到宽阱的隧穿行为.

我们测定了不同中垒厚度 $\mathrm{ADQW}$ 样品（No.40, No.60, No.80, No.200) 窄阶的光致 发光衰减过程, 实验结果如图 1 所示. 可见, 随着中垒厚度的减小, 窄陎的激子光强度衰减 加快. 由公式(1)可知, 图 1 的规律揭示出 ADQW 电子的非共振隧穿效应随中垒厚度的减少 而变强. 表 2 是各样品的隧穿时间值. 计算所用到的本征激子复合寿命 $\tau_{r}$ 选用中垒厚度 $L_{b}=200 \AA$ 时窄陎的发光衰减时间, 因为从表中看出, 当垒厚超过 $200 \AA$ 时基本观察不到隧穿

表 2 不同 $\mathrm{ADQW}$ 样品窄拼的各种时间参数

\begin{tabular}{c|c|c}
\hline 中垒曆度 $L_{b}$ & $\begin{array}{c}\text { 衰减时间 } \\
(\mathrm{ps})\end{array}$ & $\begin{array}{c}\text { 隧穿时间 } \\
(\mathrm{ns})\end{array}$ \\
\hline 40 & 58 & $67 \times 10^{-3}$ \\
60 & 340 & 17 \\
80 & 418 & 25 \\
200 & 425 & 1 \\
\hline
\end{tabular}




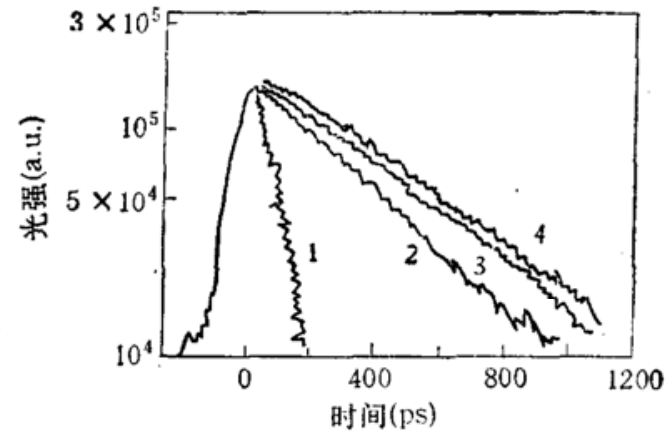

图 1 各种中垒厚度的 $\mathrm{ADQW}$ 窄阱的半对 数发光强度衰减曲线

1 为 No. 40, 2 为 No. 60, 3 为 No. 80,4 为 No. 200

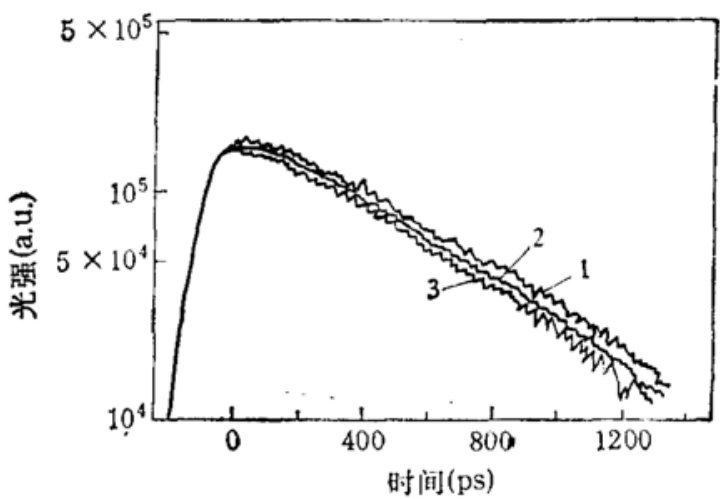

图 2 不同掺杂样品窄阱的半对数 发光强度衰减曲线

1 为 No. 601,2 为 No. 602,3 为 No. 603

过程.

此外, 还测量了不掺杂和多种掺杂方式样品窄陫的时间分辨光致发光. 图 2 是这些样品 的半对数发光强度衰减实验曲线. 从图中曲线得到的激子衰减时间分别为: No.601, $\tau-$

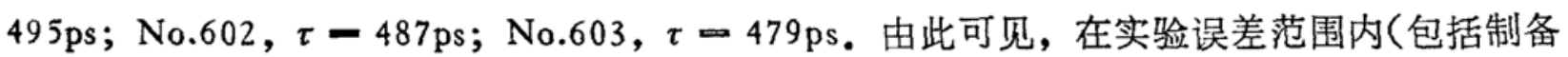
条件和样品参数), 三个样品的衰减时间可认为基本相同, 与掺杂与否以及掺杂的方式均无关.

这一实验结果显然与相干隧穿机制所预期的结果不符. 因为中垒 $\delta$ 掺杂和一般掺 $\mathrm{Be}$ 会导 致载流子受到杂质的散射增大,使载流子波函数相干性受到更大的破坏. 按照相干隧穿模型, 必然得出中垒掺杂可以减弱隧穿效应, 使窄阱激子衰减时间增大的结论. 但现在事实却并非 如此, 说明了相干隧穿模型并不成立. 另外, 以上的实验结果同样也排除了杂质散射辅助型隧 穿机制. 对于杂质散射辅助型隧穿机制, 载流子隧穿的快慢与中垒的掺杂情况密切相关. 掺 杂样品载流子受到杂质散射的几率远大于不掺杂样品, 掺杂后窄阱载流子的隧窄变快, 发光衰 减时间减小. 这与实验不符, 从而排除了这一机制.

最后, 我们初步提出一个新的激子束缚型载流子隧穿模型. 量子阱在低温下光致发光以 激子复合为主要机制 ${ }^{[12]}$, 而并非自由电子的复合. 因此实验观察到的窄阱激子发光寿命与垒 厚密切相关的现象说明了隧穿所涉及的电子或空穴不是纯自由电子或空穴, 而是已结合成激 子的电子或空穴部分, 实验观察到的隧穿过程实质上是一种激子束缚型的载流子隧穿过程. 由 $\mathrm{ADQW}$ 能量本征态的量子力学处理可知, 定域在窄咞的激子态中的电子或空穴波函数部 分仍有一定的几率分布在宽阱里. 当宽阱存在“有效”的弛豫途径, 能极快衰减从窄阱穿透人 宽阱的电子或空穴部分时, 窄陎激子态发生离解, 从而出现了所观察到的隧穿现象. 所谓“有 效”, 是指宽阱弛豫途径的电子或空穴衰减速率远大于窄阱激子的复合速率. 隧穿速率正比于 窄阱波函数在宽阱的分布几率与弛豫途径衰减速率的乘积. 根据这一模型, 不仅可以较好地 解释目前已有的隧穿实验结果, 而且还能导出有关隧穿时间下限的结论以及揭示出一种新的 较慢的共振隧穿过程. 限于篇幅, 我们将另文讨论.

\section{参考文献}

[1] Tsu, R., Esaki, L., Appl. Phys. Lett., 1973, 22: 562.

[2] Lutyi, S., Solid State Commun., 1988, 65: 787. 
[3] Capasso, F., Mohammed, K., Cho, A. Y., Appl. Phys. Lett., 1986, $48: 478$.

[ 4 ] Masumoto, Y., Tarucha, S., Okamoto, H., Phys, Rev, B, 1986, 33: 5981.

[5] Oberli, D. Y. et al, Phys. Rev., B, 1989, 40: 3028.

[6] Sawaki, N., Huptel, R. A., Gornik, E. et al., Appl. Phys. Lett, 1989, 55: 1996.

[ 7 ] Alexander, M. G. W., Nido, M., Ruhle, W. W. et al., Phys. Rev., B, 1990, 41: 12295:

[8] Matsuse, T., Tsuchiya, M., Schulman, J. N. et al., Phys. Rev. B, 1990, 42: 5719.

[ 9 ] Nido, M., Alexander, M. G. W., Ruhle, W. W. et al, Phys. Rev, B, 1991, 43: 1839.

[10] Leo, K. et al., Phys. Rev. Lett, 1991, 66: 201.

[11] 黄坥光等,物理学报, $1991,40: 1677$.

[12]崔捷、黄旭光、蔡志岗等,科学通报,1991,36(24): 1853. 